\title{
ERRATUM: "POLARIMETRIC IMAGING OF LARGE CAVITY STRUCTURES IN THE PRE-TRANSITIONAL PROTOPLANETARY DISK AROUND PDS 70: OBSERVATIONS OF THE DISK” (2012, ApJL, 758, L19)
}

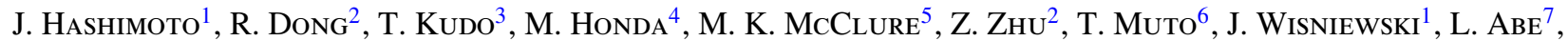

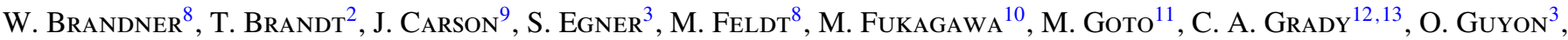

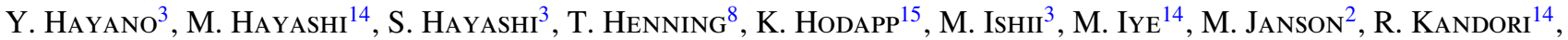 \\ G. Knapp ${ }^{2}$, N. Kusakabe ${ }^{14}$, M. Kuzuhara ${ }^{14,16,17}$, J. Kwon ${ }^{14,18}$, T. Matsuo ${ }^{19}$, S. Mayama ${ }^{20}$, M. W. McElwain ${ }^{12}$, \\ S. Miyama ${ }^{21}$, J.-I. Morino ${ }^{14}$, A. Moro-Martin ${ }^{2,22}$, T. Nishimura ${ }^{3}$, T.-S. Pyo ${ }^{3}$, G. Serabyn ${ }^{23}$, T. Suenaga ${ }^{14,18}$, H. Suto ${ }^{14}$, \\ R. Suzuki ${ }^{14}$, Y. Takahashi ${ }^{14,24}$, M. Takami ${ }^{25}$, N. Takato ${ }^{3}$, H. Terada ${ }^{3}$, C. Thalmann ${ }^{26}$, D. Tomono ${ }^{3}$, E. L. Turner ${ }^{2,27}$,

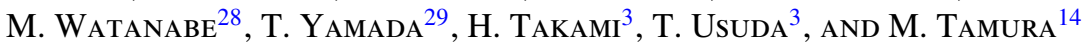 \\ ${ }^{1}$ H. L. Dodge Department of Physics and Astronomy, University of Oklahoma, 440 West Brooks St Norman, OK 73019, USA; jun.hashimoto@ ou.edu \\ ${ }^{2}$ Department of Astrophysical Sciences, Princeton University, NJ 08544, USA \\ ${ }^{3}$ Subaru Telescope, 650 North A'ohoku Place, Hilo, HI 96720, USA \\ ${ }^{4}$ Kanagawa University, 2946 Tsuchiya, Hiratsuka, Kanagawa 259-1293, Japan \\ ${ }^{5}$ Department of Astronomy, The University of Michigan, 500 Church St., 830 Dennison Bldg., Ann Arbor, MI 48109, USA \\ ${ }^{6}$ Division of Liberal Arts, Kogakuin University, 1-24-2, Nishi-Shinjuku, Shinjuku-ku, Tokyo 163-8677, Japan \\ ${ }^{7}$ Laboratoire Hippolyte Fizeau, UMR6525, Universite de Nice Sophia-Antipolis, 28, avenue Valrose, F-06108 Nice Cedex 2, France \\ ${ }^{8}$ Max Planck Institute for Astronomy, Heidelberg, Germany \\ ${ }^{9}$ Department of Physics and Astronomy, College of Charleston, 58 Coming St., Charleston, SC 29424, USA \\ ${ }^{10}$ Osaka University, 1-1, Machikaneyama, Toyonaka, Osaka 560-0043, Japan \\ ${ }^{11}$ Universitäts-Sternwarte München Scheinerstr. 1, D-81679 Munich, Germany \\ ${ }^{12}$ Exoplanets and Stellar Astrophysics Laboratory, Code 667, Goddard Space Flight Center, Greenbelt, MD 20771, USA \\ ${ }_{13}$ Eureka Scientific, 2452 Delmer, Suite 100, Oakland, CA 96002, USA \\ ${ }^{14}$ National Astronomical Observatory of Japan, 2-21-1 Osawa, Mitaka, Tokyo 181-8588, Japan \\ ${ }^{15}$ University of Hawaii, 640 North A'ohoku Place, Hilo, HI 96720, USA \\ ${ }^{16}$ Department of Earth and Planetary Science, University of Tokyo, 7-3-1 Hongo, Tokyo 113-0033, Japan \\ ${ }^{17}$ Department of Earth and Planetary Sciences, Tokyo Institute of Technology, 2-12-1 Ookayama, Meguro-ku, Tokyo 152-8551, Japan \\ ${ }^{18}$ Department of Astronomical Science, Graduate University for Advanced Studies (Sokendai), Tokyo 181-8588, Japan \\ ${ }^{19}$ Department of Astronomy, Kyoto University, Kita-shirakawa-Oiwake-cho, Sakyo-ku, Kyoto 606-8502, Japan \\ ${ }^{20}$ The Graduate University for Advanced Studies, Shonan International Village, Hayama-cho, Miura-gun, Kanagawa 240-0193, Japan \\ ${ }^{21}$ Hiroshima University, 1-3-2 Kagamiyama, Higashi-Hiroshima 739-8511, Japan \\ ${ }^{22}$ Department of Astrophysics, CAB-CSIC/INTA, E-28850 Torrej’on de Ardoz, Madrid, Spain \\ 23 Jet Propulsion Laboratory, California Institute of Technology, 4800 Oak Grove Drive, Pasadena, CA 91109, USA \\ ${ }^{24}$ Department of Astronomy, The University of Tokyo, 7-3-1 Hongo, Bunkyo-ku, Tokyo 113-0033, Japan \\ ${ }^{25}$ Institute of Astronomy and Astrophysics, Academia Sinica, P.O. Box 23-141, Taipei 10617, Taiwan \\ ${ }^{26}$ Astronomical Institute “Anton Pannekoek," University of Amsterdam, Postbus 94249, 1090 GE, Amsterdam, The Netherlands \\ ${ }^{27}$ Kavli Institute for the Physics and Mathematics of the Universe, The University of Tokyo, Kashiwa 227-8568, Japan \\ ${ }^{28}$ Department of Cosmosciences, Hokkaido University, Sapporo 060-0810, Japan \\ ${ }_{29}$ Astronomical Institute, Tohoku University, Aoba, Sendai 980-8578, Japan \\ Received 2013 August 21; published 2013 September 10
}

Online-only material: color figure

We report an error in calculating the contrast curve in the $L^{\prime}$-band LOCI image of PDS 70. In the published version of this Letter, the contrast in the bottom right panel in Figure 2 was overestimated by a factor of 10 because we measured it relative to the peak flux of the central star. The detectable mass limit for companions within the gap around PDS 70, therefore, was also overestimated by about 10 times.

We have recalculated the contrast and corrected Figure 2. The contrast was calculated relative to the photometric counts within a PSF-size circular aperture with a diameter of 5 pixels, which is a robust method for contrast calculation. Note that since we only recalculated the contrast without reprocessing the LOCI reduction, the appearance of the disk in the $L^{\prime}$-band LOCI image in Figure 2(c) is same as in the original figure. Taking into account this revision, we put an upper limit of $\sim 2$ to $\sim 4 M_{\mathrm{J}}$ on the mass of companions within the gap around PDS 70. 

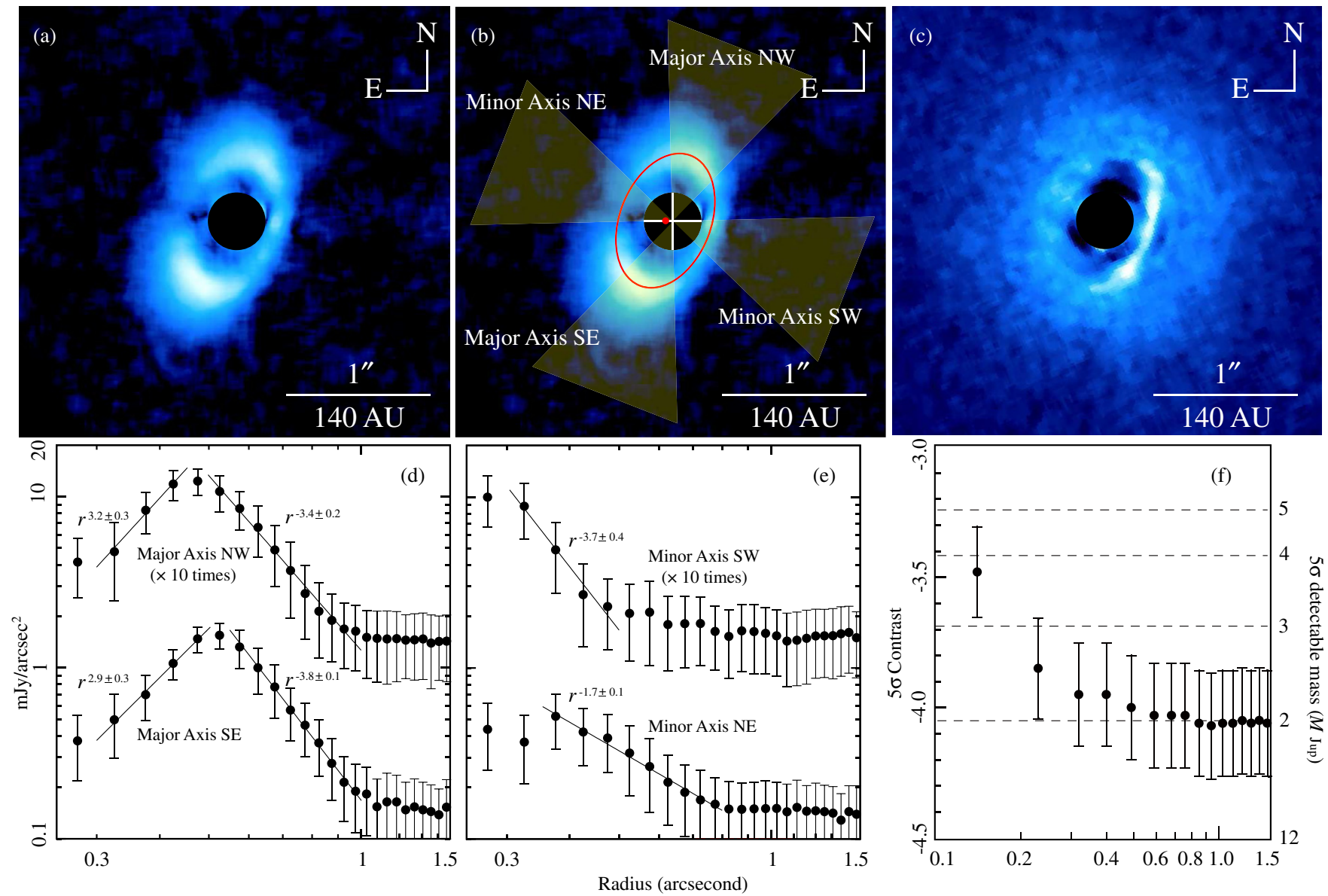

Figure 2. Observational results of PDS 70. Note that only panel (f) was revised in this Erratum. (a): $H$-band $P I$ image of PDS 70 with a software mask with $0 . \prime 4$ diameter. (b): same with (a), but its features. The solid ellipse indicate the ring-like disk. The filled circle represents the geometric center of the disk. (c): $L^{\prime}$-band LOCI image of PDS 70 with a software mask with 0'.4 diameter. The parameters in LOCI reductions are described in Section 2.2. The field of view (FOV) in the three images is $3{ }^{\prime \prime} 0 \times 33^{\prime \prime} 0$. These three images were convolved with spatial resolution. (d) and (e): radial profiles at yellow hatched regions of minor and major axes in (b). The values of the profile at northwest and southwest are multiplied by 10 for presentation. (f): detectable mass at $5 \sigma$ around PDS 70 based on the $L^{\prime}$-band LOCI image. The LOCI parameters are same with those described in Section 2.2, but the optimization area is $250 \times \mathrm{FWHM}\left(N_{\mathrm{A}}=250\right)$.

(A color version of this figure is available in the online journal.) 